\title{
Phosphorylated-myosin light chain mediates the destruction of small intestinal epithelial tight junctions in mice with acute liver failure
}

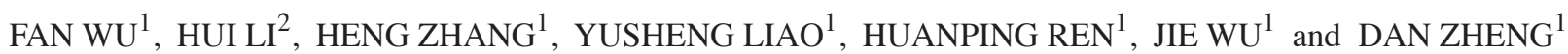 \\ ${ }^{1}$ Department of Gastroenterology, The Central Hospital of Wuhan, Tongji Medical College, Huazhong \\ University of Science and Technology, Wuhan, Hubei 430014; ${ }^{2}$ Hepatology Unit and Department of Infectious \\ Diseases, Nanfang Hospital, Southern Medical University, Guangzhou, Guangdong 510515, P.R. China
}

Received December 8, 2019; Accepted February 15, 2021

DOI: $10.3892 / \mathrm{mmr} .2021 .12031$

\begin{abstract}
Tight junction dysregulation and epithelial damage contribute to intestinal barrier loss in patients with acute liver failure (ALF); however, the regulatory mechanisms of these processes remain poorly understood. The aim of the present study was to investigate the changes of intestinal tight junction and intestinal mucosa in mice with ALF and their mechanisms. In the present study, ALF was induced in mice through an intraperitoneal injection of D-galactosamine and lipopolysaccharide (D-GalN/LPS), and the morphological changes of the liver or small intestine were analyzed using hematoxylin and eosin staining, scanning electron microscopy (SEM) and transmission electron microscopy (TEM). The intestinal tissues and isolated serum were analyzed using western blotting, immunofluorescence staining and ELISA. D-GalN/LPS-induced mice exhibited signs of hepatocyte necrosis, alongside inflammatory cell infiltration into the liver tissue and partial microvilli detachment in the small intestinal mucosa. TEM demonstrated that the intestinal epithelial tight junctions were impaired, whereas SEM micrographs revealed the presence of abnormal microvilli in D-GalN/LPS-induced mice. In addition, the expression levels of phosphorylated (p)-myosin light chain (MLC), MLC kinase (MLCK) and Rho-associated kinase (ROCK) were significantly increased in the D-GalN/LPS-induced mice compared with those in the control mice, whereas the subsequent inhibition of MLCK or ROCK significantly reduced p-MLC expression levels. Conversely, the expression levels of occludin and zonula occludens-1 (ZO-1) were significantly decreased in the D-GalN/LPS-induced mice, and the inhibition
\end{abstract}

Correspondence to: Dr Dan Zheng, Department of Gastroenterology, The Central Hospital of Wuhan, Tongji Medical College, Huazhong University of Science and Technology, 26 Shengli Street, Jiang'an, Wuhan, Hubei 430014, P.R. China

E-mail: zhengdan@zxhospital.com

Key words: myosin light chain, myosin light chain kinase, Rho-associated kinase of MLCK or ROCK significantly increased occludin and ZO-1 protein expression levels compared with those in the control group. Changes in the serum levels of tumor necrosis factor- $\alpha$ (TNF- $\alpha$ ) and interleukin (IL)-6 were similar to the trend observed in p-MLC expression levels. In conclusion, the findings of the present study suggested that in a D-GalN/LPS-induced ALF model, TNF- $\alpha$ and IL- 6 signaling may increase MLCK and ROCK expression levels, further mediate phosphorylation of MLC, which may result in tight junction dysregulation and intestinal barrier dysfunction.

\section{Introduction}

The intestinal mucosal barrier serves an important role in the development of liver failure (1); in patients with acute liver failure (ALF), the function of the intestinal barrier is compromised following severe structural damage to the mucosa (2). As a result, bacteria, lipopolysaccharide (LPS) and bacterial metabolites are more likely to invade the bloodstream, which has been shown to aggravate liver damage (3). The integrity of the intestinal barrier relies on a variety of mucosal structural components that are tightly regulated during homeostasis and disease (4). Tight junctions are one of the most important components of the intestinal mucosal barrier and serve as determinants of intestinal mucosal permeability (4). Notably, the dysfunction of tight junctions and the subsequent increased permeability have been demonstrated to facilitate the translocation of bacteria and microbial products, which induces severe inflammatory responses in the target organ (5).

A previous study reported that the intestinal mucosa tight junctions and intestinal barrier in mice with ALF were impaired (6).The major molecular pathways regulating the intestinal barrier function include the $\mathrm{Ca}^{2+} /$ myosin light chain kinase (MLCK), protein kinase C/phosphatase inhibitory protein-17 and RhoA/Rho-associated kinase (ROCK) signaling pathways (7-9); however, a previous study reported that only MLCK and ROCK were involved in the destruction of tight junctions, further regulating intestinal function (9). MLCK and ROCK are two important kinases, which phosphorylate MLC in vitro and in vivo $(10,11)$. The phosphorylation of MLC is a common intermediate in the pathophysiological regulation of intestinal barrier function; it mediates 
actin contraction and cytoskeletal rearrangements, which results in mucosal barrier dysfunction (12). However, in different cell types, MLCK- or ROCK-mediated MLC phosphorylation does not occur in an identical manner $(10,11,13)$, and the underlying mechanisms of MLC phosphorylation and its contribution to the disease pathogenesis in patients with ALF remains poorly understood. Thus, the present study aimed to investigate these processes using a mouse model of ALF.

\section{Materials and methods}

Reagents. D-galactosamine (D-GalN; cat. no. G0500) and LPS (cat. no. L2630) were purchased from Sigma-Aldrich; Merck KGaA. The MLCK inhibitor ML-7 (cat. no.S8388) and ROCK inhibitor Y-27632 (cat. no. S1049) were purchased from Selleck Chemicals. The monoclonal rabbit anti-MLCK antibody (cat. no. ab76092), monoclonal rabbit anti-ROCK antibody (cat. no. ab45171), monoclonal rabbit anti-occludin antibody (cat. no. ab167161) and polyclonal rabbit anti-zonula occludens-1 (ZO-1) antibody (cat. no. ab96587) were all purchased from Abcam. Monoclonal mouse anti-phosphorylated (p)-MLC antibody (cat. no. 3675), polyclonal rabbit anti-MLC antibody (cat. no. 3672) and the monoclonal rabbit anti-GAPDH antibody (cat. no. 2118) were purchased from Cell Signaling Technology, Inc. The polyclonal rabbit anti-p-MLC antibody (cat. no. PAB 19884) and the ELISA kits for tumor necrosis factor (TNF)- $\alpha$ (cat. no. MU30030) and interleukin (IL)-6 (cat. no. MU30044) were purchased from Bioswamp Life Science Lab.

Animal studies. As there are no facilities for keeping animals in the Central Hospital of Wuhan, Tongji Medical College Huazhong University of Science and Technology, animal experiments were conducted at Model Animal Research Institute of Wuhan Myhalic Biotechnology Co., Ltd. All animal procedures in the present study were approved by the Ethics Committee of Model Animal Research Institute of Wuhan Myhalic Biotechnology Co., Ltd. (approval no. HLK-20180621-01). Animal welfare was considered during the experiment, including efforts to minimize suffering and distress. Mice were sacrificed when they lost their appetite, $20 \%$ of their original weight, remained immobile, exhibited fast shallow breathing, demonstrated slowed responses or were listless. Male specific pathogen-free-class BALB/c mice (age, 6-8 weeks; weight, 20-22 g; $n=26$ ) were purchased from the Huazhong Agricultural University Laboratory Animal Center. The total of 26 mice included 2 extras, which were purchased as substitutes in the event of mortality. Mice were housed in pathogen-free conditions at $22^{\circ} \mathrm{C}, 50 \%$ relative humidity and under a 12 -h light/dark cycle. Mice health and behavior were monitored every $12 \mathrm{~h}$. After an initial 7 days of provision of food and water ab libitum, the mice received subsequent intervention. Mice were randomly divided into four groups ( $n=6$ mice/group), one of which served as the control group. After grouping, the remaining two mice were donated to other researchers for experiments.

Control mice were injected with PBS intraperitoneally. ALF was induced in the remaining three groups through an intraperitoneal injection of $380 \mathrm{mg} / \mathrm{kg} \mathrm{D}-$ GalN and $10 \mu \mathrm{g} / \mathrm{kg}$ LPS, of which the doses of the drugs were determined based on previous studies $(14,15)$. After 3 - and 6-h of induction, mice in each of the four groups were either injected intraperitoneally with PBS (control group), PBS (D-GalN/LPS group), $2 \mu \mathrm{g} / \mathrm{gML}-7$ (D-GalN/LPS + MLCK inhibition group) or $20 \mu \mathrm{g} / \mathrm{g}$ Y-27632 (D-GalN/LPS + ROCK inhibition group). Mice health and behavior were monitored every $4 \mathrm{~h}$ after intraperitoneal injection of D-GalN/LPS.

The mice were sacrificed with $100 \mathrm{mg} / \mathrm{kg}$ sodium pentobarbital via intraperitoneal injection $12 \mathrm{~h}$ after intraperitoneal injection of D-GalN/LPS; death was confirmed by observing respiration and by using the corneal reflection method. Blood and tissue samples were collected after the mice were sacrificed. Briefly, the abdomen was opened, the portal vein was exposed and blood was aspirated into a syringe. The blood samples were allowed to stand for 4 to $5 \mathrm{~h}$, prior to being centrifuged at $3,000 \mathrm{x} \mathrm{g}$ for $5 \mathrm{~min}$ at $4^{\circ} \mathrm{C}$ and stored at $-80^{\circ} \mathrm{C}$. The liver tissue samples were washed in PBS to remove excess blood and the samples were subsequently fixed in $40 \mathrm{~g} / 1$ paraformaldehyde (PFA) for $24 \mathrm{~h}$ at $4^{\circ} \mathrm{C}$ or frozen immediately at $-80^{\circ} \mathrm{C}$. The terminal $3 \mathrm{~cm}$ of the small intestinal tissues were also excised and fixed in $40 \mathrm{~g} / \mathrm{l} \mathrm{PFA}$ or frozen immediately at $-80^{\circ} \mathrm{C}$. It took $\sim 2$ min to collect blood and tissue samples from each mouse.

Morphological analysis using hematoxylin and eosin (H\&E) staining and scanning electron microscopy (SEM). Standard H\&E staining was performed to assess the overall morphology. Briefly, the fixed liver and small intestine tissues were fixed in $40 \mathrm{~g} / 1 \mathrm{PFA}$ at $4^{\circ} \mathrm{C}$ for $12 \mathrm{~h}$, paraffin embedded and sectioned routinely for $\mathrm{H} \& \mathrm{E}$ staining. Histomorphology was observed with a light microscope. For SEM, the small intestine tissues were fixed in $2.5 \%$ glutaraldehyde at $4^{\circ} \mathrm{C}$ for $6 \mathrm{~h}$ and $1 \%$ osmium tetroxide $\left(\mathrm{OsO}_{4}\right)$ at $4^{\circ} \mathrm{C}$ for $2 \mathrm{~h}$, dehydrated in ethanol, dried by the critical-point method and coated with a thin layer of gold. The intestinal mucosa was observed by scanning electron microscope (cat. no. SNE-3000MB; SEC).

Transmission electron microscopy (TEM). Mice were anesthetized with an intraperitoneal injection of $100 \mathrm{mg} / \mathrm{kg}(2 \%)$ sodium pentobarbital. Mice were sacrificed prior to small intestine collection. The small intestine was rapidly removed from each mouse and fixed in $2.5 \%$ glutaraldehyde. Subsequently, 1-m $\mathrm{m}^{2}$ small intestine tissue sample was obtained using a sharp blade and fixed with $2.5 \%$ glutaraldehyde in $0.1 \mathrm{M}$ PBS at $4^{\circ} \mathrm{C}$ for 2-4 h. The samples were washed three times for $10 \mathrm{~min}$ each in $\mathrm{PBS}$ and post-fixed in $1 \% \mathrm{OsO}_{4}$ at $4^{\circ} \mathrm{C}$ for $1 \mathrm{~h}$. Subsequently, sections with a thickness of $60 \mathrm{~nm}$ were dehydrated in acetone and embedded in epoxy resin 618 at $40^{\circ} \mathrm{C}$ for $4 \mathrm{~h}$. Areas of interest were selected from the semi-thin sections and stained with aqueous uranyl acetate at $4^{\circ} \mathrm{C}$ for $20 \mathrm{~min}$, followed by lead citrate at $4^{\circ} \mathrm{C}$ for $15 \mathrm{~min}$. The sections were examined using an HT-7700 transmission electron microscope (Hitachi, Ltd.).

Immunofluorescence staining. There are two major types of immunofluorescence staining methods: i) Direct immunofluorescence staining in which the primary antibody is labeled with fluorescence dye and ii) indirect immunofluorescence staining in which a secondary antibody labeled with fluorochrome is used to recognize the primary antibody; the unlabeled antibody specific for the molecule of interest is called the primary antibody. This section introduced the indirect immunofluorescence staining method to detect p-MLC. The small intestine 
tissues were paraffin-embedded, $10 \%$ formaldehyde fixed at $4^{\circ} \mathrm{C}$ for $12 \mathrm{~h}$ and sliced with a thickness of $4 \mu \mathrm{m}$. The slides were washed with PBS and underwent antigen retrieval using $0.1 \%$ trypsin at $37^{\circ} \mathrm{C}$ for $10 \mathrm{~min}$, prior to being washed three times with PBS. The slides were incubated with $3 \% \mathrm{H}_{2} \mathrm{O}_{2}$ at room temperature for $10 \mathrm{~min}$, washed three times in PBS for 3 min each and incubated with anti-p-MLC primary antibody (cat. no. PAB 19884; Bioswamp Life Science Lab.; 1:100) overnight at $4^{\circ} \mathrm{C}$. Following the primary antibody incubation, the slides were washed three times in PBS for 5 min each and incubated with an Alexa Fluor 594 goat anti-rabbit IgG secondary antibody (PAB160018, Bioswamp Life Science Lab) at a 1:200 dilution at $37^{\circ} \mathrm{C}$ for $1 \mathrm{~h}$. After a final wash with PBS, the slides were mounted using anti-fluorescence quenching sealing solution at $4^{\circ} \mathrm{C}$ for $5 \mathrm{~min}$, and stained cells were visualized using an Olympus CX41 fluorescent microscope (Olympus Corporation).

Western blotting. Total protein was extracted using $1 \%$ Triton X-100 from the small intestine tissue. Total protein was quantified using bicinchoninic acid protein concentration assay kit (Bioswamp Life Science Lab) and proteins were separated by SDS-PAGE. The concentration of protein in each lane was $20 \mu \mathrm{g}$ and $12 \%$ gel was used. The separated proteins were transferred onto polyvinylidene difluoride membranes and blocked with 5\% non-fat milk in 2,4,6-trinitrobenzenesul-phonic acid (TNBS) for $2 \mathrm{~h}$ at room temperature. The membranes were then incubated with the following primary antibodies overnight at $4^{\circ} \mathrm{C}$ : Anti-MLCK $(1: 5,000)$, anti-ROCK (1:5,000), anti-occludin (1:50,000), anti-ZO-1 (1:1,000), anti-p-MLC $(1: 1,000)$, anti-MLC $(1: 1,000)$ and anti-GAPDH $(1: 1,000)$. Following the primary antibody incubation, the membranes were washed three times for $10 \mathrm{~min}$ each in TNBS and incubated with corresponding horseradish peroxidase-conjugated secondary antibodies $(1: 1,000)$ at room temperature (goat anti-rabbit IgG, cat. no. PAB150011;goat anti-mouse IgG, cat. no. PAB150009, Bioswamp Life Science $\mathrm{Lab}$ ) for $1 \mathrm{~h}$. Protein bands were visualized using an electrochemiluminescence reagent (EMD Millipore) and a Tanon 5200 automatic chemiluminescence image analysis system (Tanon Science and Technology Co., Ltd.). Protein expression levels were analyzed using BandScan5.0 software (Glyko, Inc.).

ELISA. After the blood samples were allowed to stand for 4 to $5 \mathrm{~h}$, serum was obtained after centrifugation at $3,000 \mathrm{x} \mathrm{g}$ for $5 \mathrm{~min}$ at $4^{\circ} \mathrm{C}$. To determine the concentrations of TNF- $\alpha$ and IL- 6 in the serum, TNF- $\alpha$ and IL- 6 ELISA kits were used according to the manufacturer's protocol. The absorbance was measured using a Multiskan MS 352 microplate reader (Thermo Fisher Scientific, Inc.) at a wavelength of $450 \mathrm{~nm}$. All the samples were thawed once and assayed in triplicate.

Statistical analysis. After the pre-experiment, western blotting was repeated twice and the other tests were repeated three times. Statistical analysis was performed using SPSS 13.0 software (SPSS, Inc.) and quantitative data are presented as the mean \pm standard deviation. Statistical differences were determined using one-way ANOVA followed by Tukey's post hoc analysis. $\mathrm{P}<0.05$ was considered to indicate a statistically significant difference.

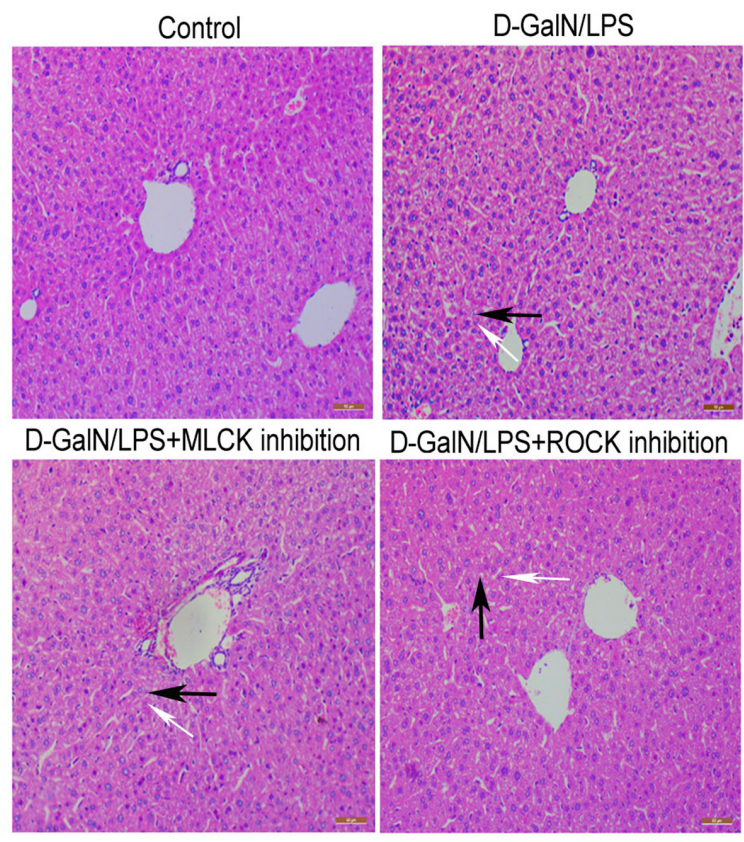

Figure 1. Representative images of the hematoxylin and eosin stained liver sections, Scale bar $=50 \mu \mathrm{m}$. Black arrows indicate inflammatory cells, white arrows indicate necrotic hepatocytes. D-GalN/LPS, D-galactosamine and lipopolysaccharide; MLCK, myosin lightchainkinase; ROCK, Rho-associated kinase.

\section{Results}

$D$-GalN/LPS induces hepatotoxicity similar to the pathology of $A L F$. D-GalN/LPS is an established model of hepatotoxicity that closely resembles clinical cases of $\operatorname{ALF}(11,12)$. In the present study, D-GalN/LPS stimulation was demonstrated to induce hepatotoxicity in mice, which was determined through the increased inflammatory cell infiltration and the accumulating presence of hepatocyte necrosis (Fig. 1). By contrast, the control mice exhibited normal liver morphology, without inflammatory cell infiltration (Fig. 1). Notably, the inhibition of MLCK with ML-7 or the inhibition of ROCK with Y-27632 markedly reduced the D-GalN/LPS-induced hepatotoxicity and inflammatory cell infiltration. These findings suggested that D-GalN/LPS induced ALF in mice, and inhibition of MLCK or ROCK may be able to reduce inflammatory cell infiltration and liver damage.

D-GalN/LPS-induced mice exhibit severely damaged intestinal mucosa. Compared with the control mice, the D-GalN/LPS-induced mice exhibited signs of severe mucosal atrophy, microvilli membrane shedding, lamina propria inflammation and edema (Fig. 2A). In addition, SEM revealed a poorly organized brush border with irregular and reduced microvilli on the intestinal surface; the surface of the microvilli was rough, uneven and incomplete (Fig. 2B). However, the inhibition of either MLCK or ROCK markedly reduced the damage to the intestinal mucosa, with less inflammatory cell infiltration and mild mucosal atrophy being observed in the D-GalN/LPS+MLCK inhibition group and the D-GalN/LPS + ROCK inhibition group compared with those in the D-GalN/LPS group (Fig. 2A and B).

Intestinal epithelial tight junctions are disrupted in $D$-GalN/LPS-induced mice. TEM analysis demonstrated that 
A
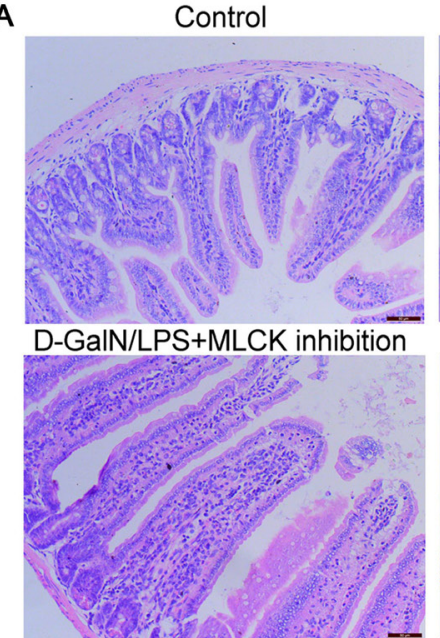

B

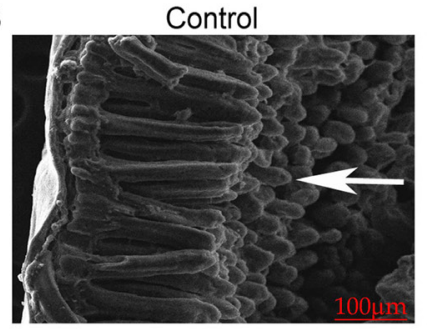

D-GalN/LPS+MLCK inhibition

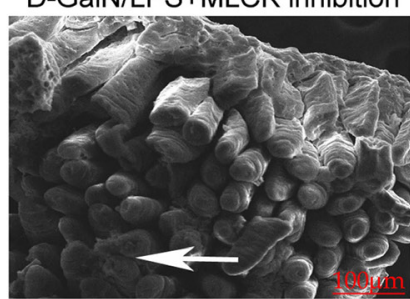

D-GalN/LPS

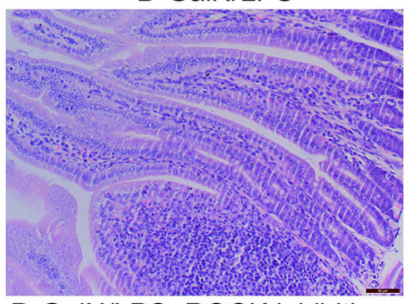

D-GaIN/LPS+ROCK inhibition

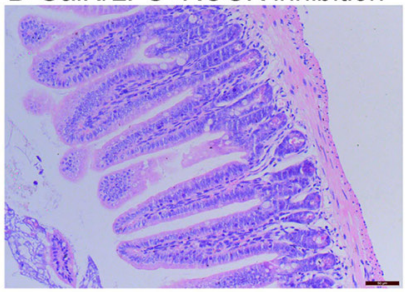

D-GaIN/LPS

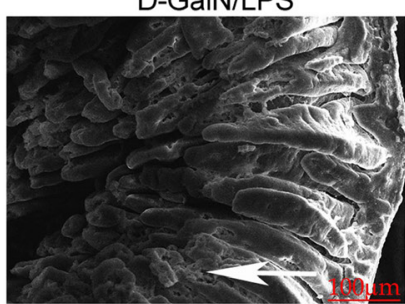

D-GalN/LPS+ROCK inhibition

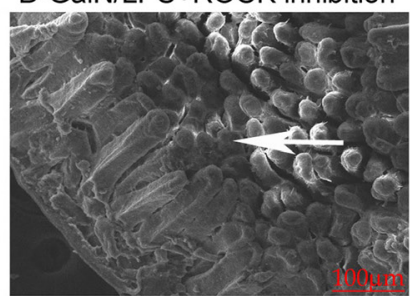

Figure 2. D-GalN/LPS-induced mice exhibit severe damage to the intestinal mucosa. (A) Representative images of hematoxylin and eosin-stained sections of the small intestine. Scale bar=50 $\mu \mathrm{m}$. (B) Representative micrographs obtained from the scanning electron microscopy of microvilli tubules. Scale bar $=100 \mu \mathrm{m}$. Arrows indicate microvilli tubules. D-GalN/LPS D-galactosamine and lipopolysaccharide; MLCK, myosin light chain kinase; ROCK, Rho-associated kinase.

the intestinal epithelial tight junctions of the control mice remained tight and intact (Fig. 3). By contrast, dilated tight junctions were observed in D-GalN/LPS-induced mice, alongside sparse, irregular microvilli with signs of partial shedding, and an expanded endoplasmic reticulum (Fig. 3). In both the D-GalN/LPS + MLCK inhibition and the D-GalN/LPS + ROCK inhibition groups, the tight junctions were revealed to be slightly dilated and the microvilli shedding was reduced compared with those in the D-GalN/LPS group (Fig. 3).

$p-M L C$ expression levels are significantly increased in D-GalN/LPS-induced mice. Immunofluorescence analysis of the intestinal tissue confirmed the presence of increased expression levels of p-MLC in the small intestine of D-GalN/LPS-induced mice, whereas the inhibition of ROCK or MLCK reduced p-MLC protein expression levels (Fig. 4A). Western blotting also confirmed that the expression levels of p-MLC were significantly increased in D-GalN/LPS-induced mice compared with those in the control mice (Fig. 4B and C). Furthermore, the expression levels of
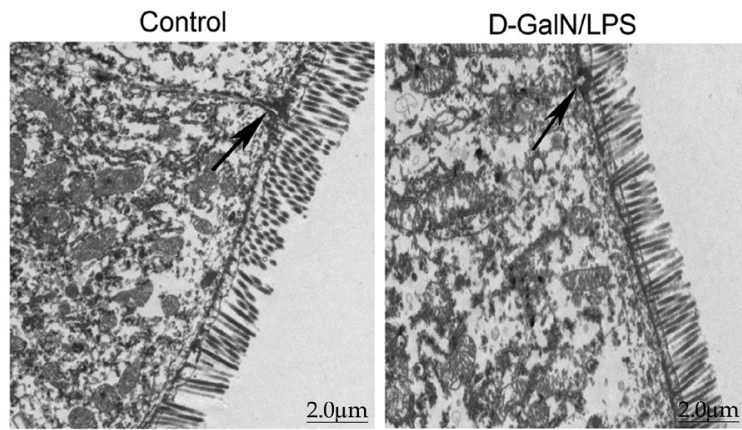

D-GaIN/LPS+MLCK inhibition
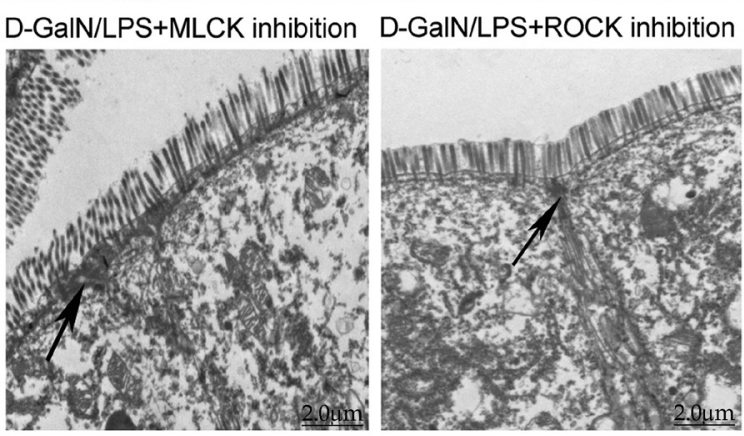

Figure 3. Tight junctions are dilated in D-GalN/LPS-induced mice. Transmission electron microscopy was used to analyze the ultrastructure of the intestine in control, D-GalN/LPS, D-GalN/LPS+MLCK inhibition and D-GalN/LPS+ROCK inhibition groups. Arrows indicate tight junctions (scale bar=2.0 $\mu \mathrm{m}$ ). D-GalN/LPS, D-galactosamine and lipopolysaccharide; MLCK, myosin light chain kinase; ROCK, Rho-associated kinase.

p-MLC in the D-GalN/LPS + MLCK inhibition group and D-GalN/LPS + ROCK inhibition group were significantly decreased compared with those in the D-GalN/LPS-treated group, which suggested that both MLCK and ROCK may be involved in regulating MLC phosphorylation (Fig. 4B and C).

Increased expression levels of MLCK and ROCK are associated with tight junction protein alternations. Since MLCK and ROCK inhibition significantly reduced the expression levels of p-MLC in the D-GalN/LPS-induced mice, it was subsequently investigated whether MLCK and ROCK expression levels were increased in these mice. As hypothesized, MLCK and ROCK expression levels were revealed to be significantly increased in the D-GalN/LPS-induced mice compared with those in the control mice (Fig. 5). The phosphorylation of MLC serves a central role in regulating barrier function, thus whether the increased p-MLC expression levels altered tight junction protein levels was investigated. Tight junction proteins include occludin and ZO-1. Therefore, the present study detected the expression levels of occludin and ZO-1. Compared to those in the control group, the protein expression levels of occludin and ZO-1 were significantly decreased in the D-GalN/LPS-induced mice (Fig. 5), whereas inhibition of MLCK and ROCK significantly reduced MLCK and ROCK expression levels, whilst simultaneously increasing occludin and ZO-1 levels (Fig. 5).

Serum TNF- $\alpha$ and IL- 6 levels are increased in $D$-GalN/LPS-induced mice. The immune response serves a pivotal role in the pathogenesis of liver failure, and the expression of inflammatory cytokines, including IL-6 and TNF- $\alpha$, has been associated with ALF (16). Thus, ELISA was 
A
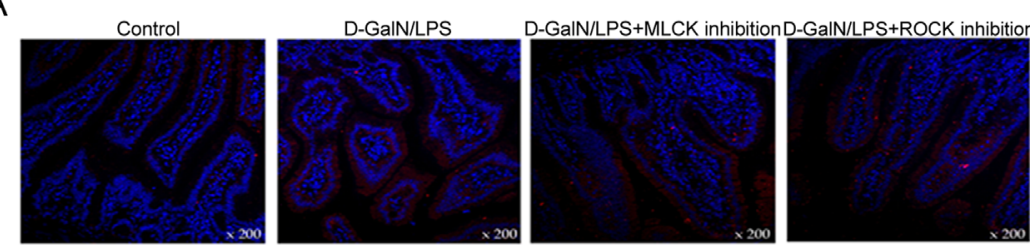

B

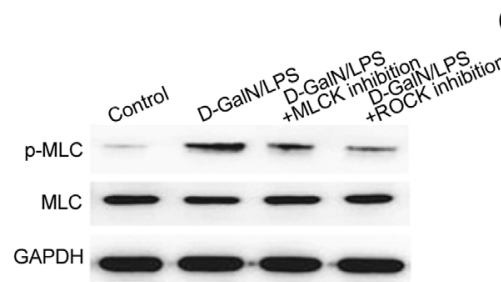

D
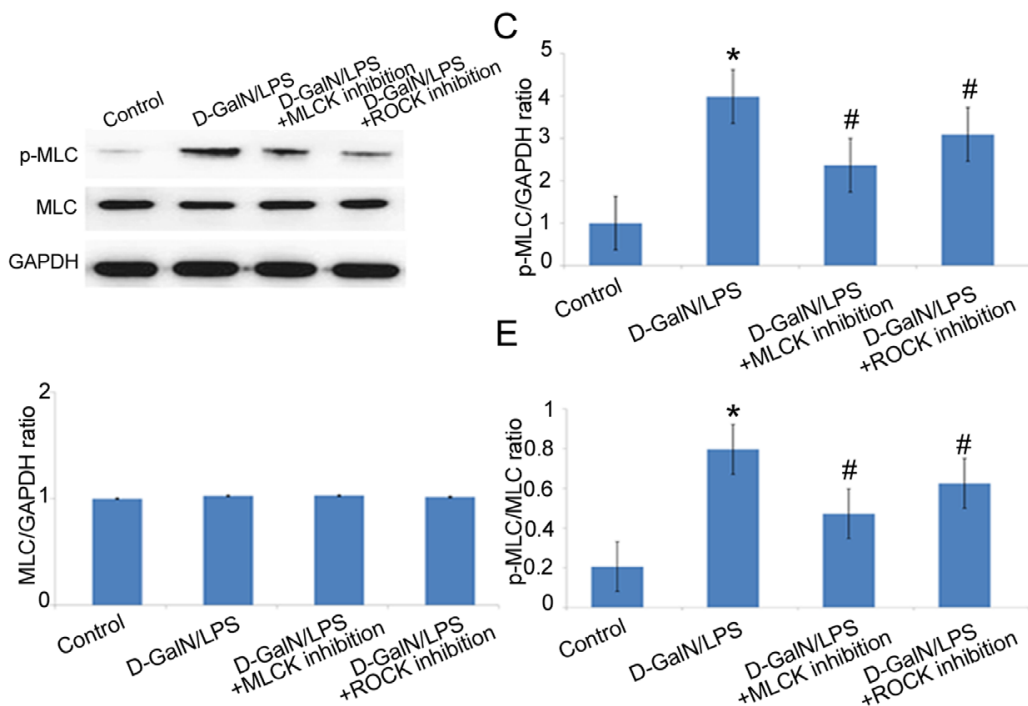

Figure 4. Expression levels of p-MLC are increased by D-GalN/LPS and decreased by MLCK or ROCK inhibition. (A) Immunofluorescence staining was used to analyze the expression level of p-MLC in the control, D-GalN/LPS, D-GalN/LPS + MLCK inhibition and D-GalN/LPS + ROCK inhibition groups; red staining indicates p-MLC, blue staining indicates the nucleus (magnification, x200). (B) Western blot analysis of intestinal expression levels of p-MLC and MLC. (C) Relative expression of p-MLC compared with GAPDH. GAPDH was used as an internal control. (D) Relative expression of MLC compared with GAPDH. (E) Relative expression of p-MLC compared with MLC. Data were analyzed by one-way ANOVA followed by Tukey's test. ${ }^{*} \mathrm{P}<0.01$ vs. the control group; ${ }^{\prime} \mathrm{P}<0.05$ vs. the D-GalN/LPS group. p-, phosphorylated; MLC, myosin light chain; D-GalN/LPS, D-galactosamine and lipopolysaccharide; MLCK, myosin light chain kinase; ROCK, Rho-associated kinase.

A

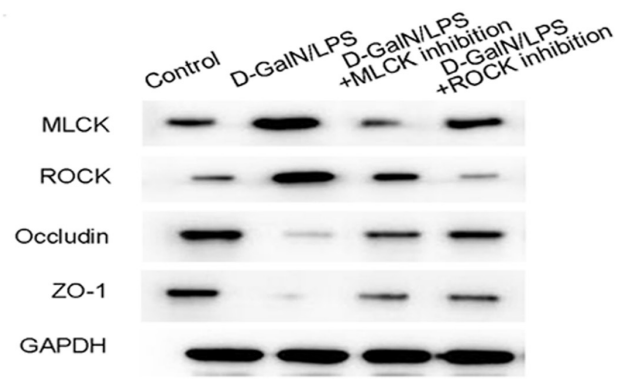

$\mathrm{B}$

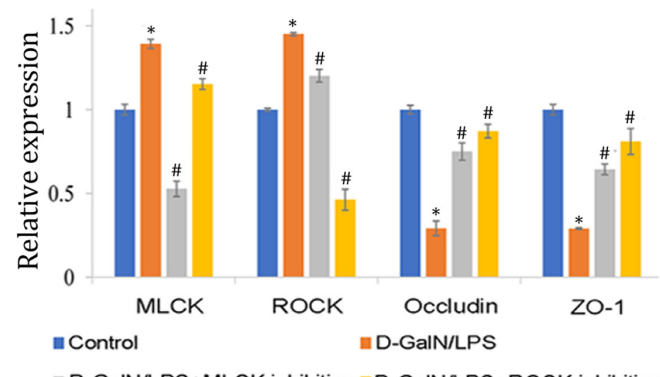

Figure 5. Tight junction protein expression levels in the control, D-GalN/LPS D-GalN/LPS + MLCK inhibition and D-GalN/LPS + ROCK inhibition groups. (A) Western blot analysis of intestinal MLCK, ROCK, occludin and ZO-1 expression levels. (B) Semi-quantification of western blotting demonstrated that the expression levels of MLCK and ROCK were significantly increased, whereas ZO-1 and occludin expression levels were significantly decreased in the D-GalN/LPS-induced mice. Data were analyzed by one-way ANOVA followed by Tukey's test. ${ }^{*} \mathrm{P}<0.01$ vs. the control group; ${ }^{*} \mathrm{P}<0.05$ vs. the $\mathrm{D}-\mathrm{GalN} / \mathrm{LPS}$ group. D-GalN/LPS, D-galactosamine and lipopolysaccharide; MLCK, myosin light chain kinase; ROCK, Rho-associated kinase; ZO-1, zonula occludens-1.

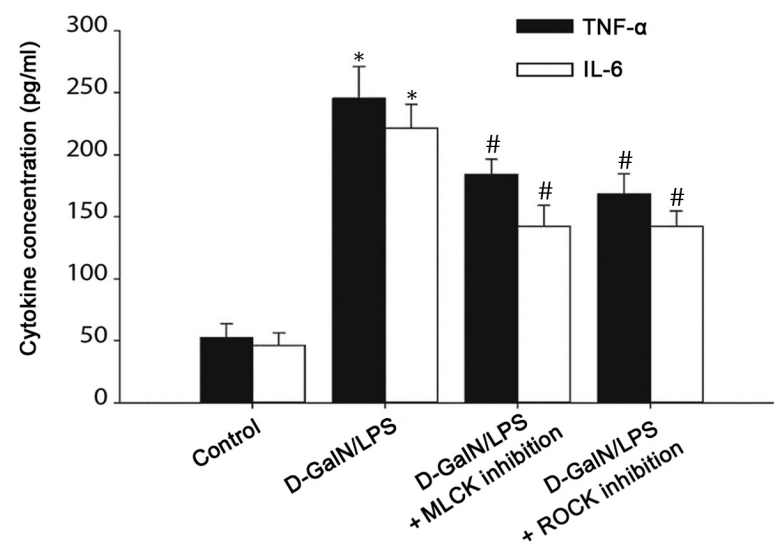

Figure 6. Serum TNF- $\alpha$ and IL-6 levels are increased in D-GalN/LPS-induced mice. The inhibition of MLCK expression levels using ML-7, or the inhibition of ROCK expression levels using Y-27632, significantly reduced serum TNF- $\alpha$ and IL-6 levels. Data were analyzed by one-way ANOVA followed by Tukey's test. ${ }^{*} \mathrm{P}<0.05$ vs. control group; ${ }^{\#} \mathrm{P}<0.05$ vs. $\mathrm{D}-\mathrm{GalN} / \mathrm{LPS}$ group. TNF; tumor necrosis factor; IL, interleukin; D-GalN/LPS, D-galactosamine and lipopolysaccharide; MLCK, myosin light chain kinase; ROCK, Rho-associated kinase.

performed to analyze the serum levels of TNF- $\alpha$ and IL- 6 in D-GalN/LPS-induced mice. The serum levels of TNF- $\alpha$ and IL-6 were significantly increased in the D-GalN/LPS-treated mice compared with those in the control mice. By contrast, the inhibition of MLCK or ROCK significantly reduced serum TNF- $\alpha$ and IL- 6 levels compared with those in the D-GalN/LPS group (Fig. 6). These data suggested that the tight 
junction dysregulation observed in the D-GalN/LPS-induced mice may be mediated through TNF- $\alpha$ and IL-6 signaling, and the inhibition of MLC phosphorylation may be able to reduce TNF- $\alpha$ and IL-6 signaling.

\section{Discussion}

Epithelial tight junctions are important structures that regulate the permeability of the intestinal epithelial barrier (4). Increased intestinal permeability often accompanies liver disease (2); however, the roles of permeability defects and intestinal barrier dysfunction in disease pathogenesis remain poorly understood. Thus, the present study aimed to define the molecular mechanisms involved in barrier dysfunction and increased permeability during disease progression.

D-GalN/LPS-induced acute liver injury is a well-established in vivo model of ALF $(17,18)$. In this model, LPS, an endotoxin, activates macrophages and Kupffer cells to produce TNF- $\alpha$, which activates a variety of cytokines that mediate cell death and inflammation (19). Meanwhile, $\mathrm{D}$-GalN is a selective hepatotoxin that depletes uridine nucleotides in the liver, which is subsequently found to inhibit RNA synthesis in hepatocytes and enhance the toxicity of TNF- $\alpha$ (20). In previous studies, it was reported that mice who received D-GalN and LPS via intraperitoneal injection to induce acute liver injury exhibited signs of rapid cell death that closely resembled clinical cases of ALF $(14,15)$. In the present study, an increased prevalence of hepatocyte necrosis and inflammatory cell infiltration was observed in the D-GalN/LPS-induced mice. The diagnosis of ALF includes ALT, AST and pathology (21). It was limitation of the present study that ALT and AST were not detected. ALT and AST detection would be more useful in the diagnosis of ALF.

The phosphorylation of MLC is a common intermediate in the pathophysiological regulation of the mucosal barrier (12). Previously, studies have reported that MLC can be phosphorylated by both MLCK and ROCK $(10,11)$; however, although both kinases share the same phosphorylation sites, each has been demonstrated to have distinct roles in the spatial regulation of MLC phosphorylation $(22,23)$. This phenomenon has been observed in different cells and species, thus suggesting that either the joint or single action of MLCK and ROCK serves the leading role depending on the cell or species type. For example, in an inflammatory bowel disease model, the activation of MLCK led to tight junction dysfunction and a dysregulated intestinal mucosal barrier in early-stage disease, whereas in the advanced stages, the loss of tight junction function was revealed to be independent of MLCK (24). During the mycophenolic acid-mediated barrier function test of the human colon cancer cell line Caco-2,MLC and MLCK were also discovered to be involved in the destruction of tight junctions and changes in the cell permeability (25); however, whether ROCK was involved in the destruction of the tight junctions was unclear. It is well established that the intestinal barrier is dynamically regulated (26). The present study demonstrated that in ALF model mice, the expression levels of p-MLC were significantly increased, and both MLCK and ROCK were suggested to be involved in the phosphorylation of MLC, because the inhibition of either of them significantly reduced p-MLC expression levels. However, the differences between the MLCK/ROCK inhibition groups and the control group suggested that both may be required or that other signaling pathways may also be involved.

Cytokines are critical for intercellular communication and mucosal homeostasis, and they are also important drivers of intestinal inflammation and mucosal damage (27). Thus, it was hypothesized that the inflammatory factors TNF- $\alpha$ and IL-6 may initially trigger tight junction dysregulation. For example, a previous study reported that the morphological abnormalities in the intestinal mucosa of ALF mice were positively associated with serum TNF- $\alpha$ levels, whereas TNF- $\alpha$ antibodies significantly prevented changes in the intestinal tissue ultrastructure and ZO-1 expression (6). Unfortunately, this previous study did not investigate the mechanisms of intestinal mucosal damage and tight junction molecular changes (6). In addition, anti-TNF- $\alpha$ antibody therapy is widely used to treat Crohn's disease and ulcerative colitis, demonstrating its important role in regulating intestinal permeability $(28,29)$. There are several lines of evidence indicating the effect of IL- 6 on paracellular permeability; for example, in IL-6-knockout mice, the increased intestinal permeability to small molecules was linked to the stability of ZO-1 in tight junctions (30). In addition, IL- 6 treatment increased the permeability across endothelial cells through inducing the aberrant localization of ZO-1 and actin structure remodeling, of which these effects were reversible (31). The present study demonstrated that TNF- $\alpha$ and IL- 6 levels were significantly increased in the ALF model mice; unfortunately, other cytokines were not tested in the present study, such as IL-1,IL-8 and IL-12. Notably, the inhibition of MLCK or ROCK significantly reduced serum levels of TNF- $\alpha$ and IL-6, and prevented tight junction dysregulation. These data suggested that in ALF model mice, MLCK and ROCK signaling may be mediated by TNF- $\alpha$ and IL- 6 . However, the manner in which cytokines activate MLCK and ROCK remains largely unclear. A previous study suggested that TNF- $\alpha$ induces increases in intestinal permeability, which contributes to inflammation in disease conditions; this was mediated through NF- $\kappa B$ activation, involving the NF- $\kappa B$ p50/p65 dimer binding to the MLCK promoter region (32). A comprehensive understanding of the signaling cascade during disease progression will be the focus of future studies, including immunofluorescence analysis to detect occludin, ZO-1 and other tight junction proteins for further elucidation of tight junction function.

In conclusion, the findings of the present study suggested that D-GalN/LPS treatment may induce ALF, which was accompanied by severe damage to the intestinal mucosa and barrier function. The signal transduction pathways that regulate intestinal barrier dysfunction were also investigated, and both MLCK and ROCK were discovered to be responsible for MLC phosphorylation in the ALF model mice. Increased TNF- $\alpha$ and IL-6 levels due to liver damage were also demonstrated to be a possible trigger for mucosal damage, which led to increased intestinal permeability. Thus, targeting both MLCK and ROCK may be therapeutically effective to stabilize barrier function and reduce bacterial translocation in patients with ALF.

\section{Acknowledgements}

Not applicable. 


\section{Funding}

This work was supported by the Natural Science Foundation of Hubei Province (grant no. 2016CFB357).

\section{Availability of data and materials}

The datasets used during the present study are available from the corresponding author on reasonable request.

\section{Authors' contributions}

FW and DZ conceived and designed the study. FW, HL, HZ, YL, JW and HR acquired and analyzed the data. FW, JW and DZ confirmed the authenticity of all the raw data. FW prepared the draft of the manuscript, including the figures. All authors read and approved the final manuscript.

\section{Ethics approval and consent to participate}

All animal procedures in the present study were approved by the Ethics Committee of Model Animal Research Institute of Wuhan Myhalic Biotechnology Co., Ltd. (approval no. HLK-20180621-01).

\section{Patient consent for publication}

Not applicable.

\section{Competing interests}

The authors declare that they have no competing interests.

\section{References}

1. Vancamelbeke $M$ and Vermeire S: The intestinal barrier: A fundamental role in health and disease. Expert Rev Gastroenterol Hepatol 11: 821-834, 2017

2. Aguirre Valadez JM, Rivera-Espinosa L, Mendez-Guerrero O, Chavez-Pacheco JL, Juarez IG and Torre A: Intestinal permeability in a patient with liver cirrhosis. TherClin Risk Manag 12 1729-1748, 2016

3. Wiest R, Lawson M and Geuking M: Pathological bacterial translocation in liver cirrhosis. J Hepatol 60: 197-209, 2014.

4. Odenwald MA and Turner JR: The intestinal epithelial barrier: A therapeutic target? Nat Rev Gastroenterol Hepatol 14: 9-21, 2017.

5. Kelly JR, Kennedy PJ, Cryan JF, Dinan TG, Clarke G and Hyland NP: Breaking down the barriers: The gut microbiome, intestinal permeability and stress-related psychiatric disorders. Front Cell Neurosci 14: 392, 2015.

6. Song HL, Lv S and Liu P: The roles of tumor necrosis factor-alpha in colon tight junction protein expression and intestinal mucosa structure in a mouse model of acute liver failure. BMC Gastroenterol 9: $70,2009$.

7. Murthy KS: Signaling for contraction and relaxation in smooth muscle of the gut. Annu Rev Physiol 68: 345-374, 2006.

8. Rattan S, Phillips BR and Maxwell PJ 4th: RhoA/Rho-kinase: Pathophysiologic and therapeutic implications in gastrointestinal smooth muscle tone and relaxation. Gastroenterology 138 13-18.e1-3, 2010

9. Zolotarevsky Y, Hecht G, Koutsouris A, Gonzalez DE, Quan C, Tom J, Mrsny RJ and Turner JR: A membrane-permeant peptide that inhibits MLC kinase restores barrier function in in vitro models of intestinal disease. Gastroenterology 123: 163-172, 2002

10. Rattan S: Ca2+/calmodulin/MLCK pathway initiates, and RhoA/ROCK maintains, the internal anal sphincter smooth muscle tone. Am J Physiol Gastrointest Liver Physiol 312: G63-G66, 2017.
11. Xiong Y, Wang C, Shi L, Wang L, Zhou Z, Chen D, Wang J and Guo H: Myosin light chain kinase: A potential target for treatment of inflammatory diseases. Front Pharmacol 8: 292, 2017.

12. Cunningham KE and Turner JR: Myosin light chain kinase Pulling the strings of epithelial tight junction function. Ann N Y Acad Sci 1258: 34-42, 2012

13. Rigor RR, Shen Q, Pivetti CD, Wu MH and Yuan SY: Myosin light chain kinase signaling in endothelial barrier dysfunction. Med Res Rev 33: 911-933, 2013

14. Zhang Z, Tian L and Jiang K: Propofol attenuates inflammatory response and apoptosis to protect d-galactosamine/lipopolysaccharide induced acute liver injury via regulating TLR4/NF-кB/NLRP3 pathway. Int Immunopharmacol 77: 105974, 2019.

15. Liu X, Wang T, Liu X, Cai L, Qi J, Zhang P and Li Y: Biochanin A protects lipopolysaccharide/D-galactosamine-induced acute liver injury in mice by activating the Nrf2 pathway and inhibiting NLRP3 inflammasome activation. Int Immunopharmacol 38 324-331, 2016.

16. Simpson KJ, Henderson NC, Bone-Larson CL, Lukacs NW, Hogaboam CM and Kunkel SL: Chemokines in the pathogenesis of liver disease: So many players with poorly defined roles. Clin Sci (Lond) 104: 47-63, 2003.

17. Rietschel ET, Kirikae T, Schade FU, Mamat U, Schmidt G, Loppnow H, Ulmer AJ, Zähringer U, Seydel U, Di Padova F, et al: Bacterial endotoxin: Molecular relationships of structure to activity and function. FASEB J 8: 217-225, 1994.

18. Belanger M and Butterworth RF: Acute liver failure: A critical appraisal of available animal models. Metab Brain Dis 20: 409-423, 2005

19. Kawaratani H, Tsujimoto T, Douhara A, Takaya H, Moriya K, Namisaki T, Noguchi R, Yoshiji H, Fujimoto M and Fukui $\mathrm{H}$ : The effect of inflammatory cytokines in alcoholic liver disease. Mediators Inflamm 2013: 495156, 2013.

20. Silverstein R: D-galactosamine lethality model: Scope and limitations. J Endotoxin Res 10: 147-162, 2004.

21. Wang Y, Gao LN, Cui YL and Jiang HL: Protective effect of danhong injection on acute hepatic failure induced by lipopolysaccharide and d-galactosamine in mice. Evid Based Complement Alternat Med 2014: 153902, 2014.

22. Totsukawa G, Yamakita Y, Yamashiro S, Hartshorne DJ, Sasaki Y and Matsumura F: Distinct roles of ROCK (Rho-kinase) and MLCK in spatial regulation of MLC phosphorylation for assembly of stress fibers and focal adhesions in $3 \mathrm{~T} 3$ fibroblasts. J Cell Biol 150: 797-806, 2000

23. Kassianidou E, Hughes JH and Kumar S: Activation of ROCK and MLCK tunes regional stress fiber formation and mechanics via preferential myosin light chain phosphorylation. Mol Biol Cell 28: 3832-3843, 2017

24. Su L, Nalle SC, Shen L, Turner ES, Singh G, Breskin LA, Khramtsova EA, Khramtsova G, Tsai PY, Fu YX, et al: TNFR2 activates MLCK-dependent tight junction dysregulation to cause apoptosis-mediated barrier loss and experimental colitis. Gastroenterology 145: 407-415, 2013.

25. Qasim M, Rahman h, Ahmed R, Oellerich M and Asif AR: Mycophenolic acid mediated disruption of the intestinal epithelial tight junctions. Exp Cell Res 322: 277-289, 2014.

26. Nusrat A, Turner JR and Madara JL: Molecular physiology and pathophysiology of tight junctions. IV. Regulation of tight junctions by extracellular stimuli: Nutrients, cytokines, and immune cells. Am J Physiol Gastrointest Liver Physiol 279: G851-G857, 2000.

27. Andrews C, McLean MH and Durum SK: Cytokine tuning of intestinal epithelial function. Front Immunol 9: 1270, 2018.

28. Gibson PR: Increased gut permeability in Crohn's disease: Is TNF the link? Gut 53: 1724-1725, 2004.

29. Sands BE and Kaplan GG: The role of TNFalpha in ulcerative colitis. J Clin Pharmacol 47: 930-941, 2007.

30. Yang R, Han X, Uchiyama T, Watkins SK, Yaguchi A, Delude RL and Fink MP: IL-6 is essential for development of gut barrier dysfunction after hemorrhagic shock and resuscitation in mice. Am J Physiol Gastrointest Liver Physiol 285: G621-G629, 2003.

31. Maruo N, Morita I, Shirao M and Murota S: IL-6 increases endothelial permeability in vitro. Endocrinology 131: 710-714, 1992

32. Ye $X$ and Sun $M$ : AGR2 ameliorates tumor necrosis factor- $\alpha$-induced epithelial barrier dysfunction via suppression of NF- $\kappa \mathrm{B}$ p65-mediated MLCK/p-MLC pathway activation. Int J Mol Med 39: 1206-1214, 2017.

This work is licensed under a Creative Commons Attribution-NonCommercial-NoDerivatives 4.0 International (CC BY-NC-ND 4.0) License. 